\title{
Early Onset of Hepatosplenic T-cell Lymphoma Following Azathioprine Cessation in Ulcerative Colitis: A Case Report
}

\author{
Astrid-Jane Greenup ${ }^{1 *}$, Greg Rosenfeld ${ }^{1}$ and Mikameh Kazem ${ }^{2}$ \\ ${ }^{1}$ Division of Gastroenterology, University of New South Wales, Sydney, NSW, Australia \\ ${ }^{2}$ Department of Medicine, University of British Columbia, Canada
}

*Corresponding author: Astrid-Jane Greenup, Division of Gastroenterology, University of New South Wales, Sydney, NSW, Australia, Tel: +61477021884; E-mail: astrid_jg@hotmail.com

\begin{abstract}
Hepatosplenic T Cell Lymphoma (HSTCL) is a rare but deadly form of non-Hodgkin's lymphoma which is increasingly reported in the context of chronic immunosuppression in patients with solid organ transplant, lymphoproliferative disorder, or inflammatory bowel disease (IBD). It is of note that there is an association between the development of HSTCLand the use of thiopurines or anti-tumor-necrosis alpha inhibitors (anti-TNFs) in patients with IBD. The development of HSTCL is usually concurrent with the use of a thiopurine and an anti-TNF. We report a case of a young male with extensive Ulcerative Colitis who developed HSTCL eight months following cessation of azathioprine monotherapy. This case highlights the need for consideration of potential significant adverse side effects such as HSTCL when prescribing azathioprine, particularly in the described demographic of a young male. Furthermore, the risk for HSTCL can persist despite cessation of azathioprine.
\end{abstract}

Keywords: Hepatosplenic T cell lymphoma; Non-Hodgkin's lymphoma; Organ transplant; Anti-TNFs; Azathioprine

Received Date: January 21, 2018; Accepted Date: February 07, 2018; Published Date: February 14, 2018

\section{Introduction}

Hepatosplenic T cell lymphoma (HSTCL) is a rare but aggressive form of non-Hodgkin's lymphoma (NHL) which is often resistant to conventional therapy $[1,2]$. The reported manifestations are often hepatosplenomegaly caused by infiltration of such organs by malignant $\mathrm{T}$-cell clones, thrombocytopenia and systemic symptoms such as weight loss and fever $[2,3]$. The malignant $\mathrm{T}$-cells have distinct cell surface protein signatures, while cytogenetic studies show isochromosome 7q and trisomy of chromosome 82 .

Incidence of HSTCL has been reported to be highest in young males with inflammatory bowel disease (IBD) who have been treated with thiopurines. IBD patients treated with azathioprine or 6-mercaptopurine (6-MP) have reportedly a four-fold increase in the risk of developing lymphoma [4].

Citation: Greenup AJ, Rosenfeld G and Kazem M. Early onset of Hepatosplenic T-cell Lymphoma Following Azathioprine Cessation in Ulcerative Colitis: a Case Report. J Clin Cases Rep 2018: 1(1) 1-5. DOI: https://doi.org/10.46619/joccr.2018.1-1001 
www.tridhascholars.org | April-2018

While most of the cases are found to be NHL of the B-cell lineage, a clear association has been shown between HSTCL and exposure to thiopurines, or a combination of thiopurines and anti-TNFs $[3,5,6]$. While the exact molecular pathophysiology of thiopurines and HSTCL is the subject of many discussions, one suggested hypothesis is inhibition of immune-surveillance by cytotoxic T cells and Natural Killer cells. This is thought to be the result of incorporation of 6-thioguanine - the metabolite of both azathioprine and 6-mercaptopurine into ribonucleotides and halting protein synthesis and cell proliferation [7]. Other groups have suggested direct interaction of these antimetabolites with activated $\mathrm{T}$ cells and changing a costimulatory signal to an apoptosis signal [8]. Interestingly, Epstein-Barr virus is reported to be found in association with non-hepatosplenic gammadelta-T-cell lymphomas as opposed to HSTCL [9].

Here we report a case of a 26-year-old male with Ulcerative Colitis (UC) who developed HSTCL eight months after cessation of Azathioprine monotherapy.

\section{Case Report}

A 26-year-old male with extensive UC presented with a six-week history of fatigue, dyspnoea on exertion, weight loss and night sweats. His diagnosis of UC had occurred five years earlier, for which initial therapy was prednisone and oral mesalazine $4.8 \mathrm{~g}$ daily. Given the development of steroid dependency, 22 months into his disease course, he commenced azathioprine 150 $\mathrm{mg}$ daily (approximately a $2 \mathrm{mg} / \mathrm{kg}$ dose) with subsequent obtainment of symptomatic remission. Mesalazine was ceased one month following azathioprine commencement. Laboratory monitoring after commencement of azathioprine was unremarkable, with his most recent laboratory results while remaining on azathioprine demonstrating a normal complete blood count (CBC) with hemoglobin (Hb $148 \mathrm{~g} / \mathrm{L})$, white blood cell (WBC $\left.7.8 \times 10^{9} / \mathrm{L}\right)$, red blood cell $\left(\mathrm{RBC} 4.4 \times 10^{12} / \mathrm{L}\right)$ and platelet counts $(\mathrm{PLT}$ $\left.224 \times 10^{9} / \mathrm{L}\right)$. Renal and liver function tests were also normal.

There was no additional significant medication history. He smoked marijuana for recreational purposes and denied nicotine or alcohol use. There was no family history of IBD or hematological disorders. He worked in a gymnasium. Epstein-Barr virus status was unknown.

In the setting of prolonged symptomatic remission of 26 months while on azathioprine, he underwent a colonoscopy which demonstrated very mild distal colitis. Azathioprine was ceased given concerns with long-term use and topical amino salicylate therapy (mesalazine $4 \mathrm{~g}$ enema twice weekly) was commenced and continued for three months. Repeat laboratory examination two months following azathioprine cessation again demonstrated a normal CBC, liver enzymes and renal function.

Eight months after cessation of azathioprine, he reported a six-week history of increasing fatigue, reduced exercise tolerance, palpitations, dizziness, weight loss and night sweats. Clinical examination was significant for splenomegaly. Repeat laboratory work showed a new macrocytic anemia (Hb $85 \mathrm{~g} / \mathrm{L}$ ) and PLT count of $122 \times 10^{9} / \mathrm{L}$ with an otherwise normal WBC. Additional findings were an elevated reticulocyte count of 209, INR 1.6, lactate dehydrogenase $209 \mathrm{U} / \mathrm{L}$, bilirubin $24 \mu \mathrm{mol} / \mathrm{L}$, haptoglobin level less than $0.07 \mathrm{~g} / \mathrm{L}$, ferritin 14456 and an isolated aspartate aminotransferase elevation of $58 \mathrm{U} / \mathrm{L}$. Blood film showed a leucoerythroblastic picture.

A CT abdomen demonstrated hepatosplenomegaly with a maximal splenic diameter of $18.7 \mathrm{~cm}$, and no additional adenopathy (Figure 1). A bone marrow biopsy was conducted with findings of a hypercellular marrow, smudging and an infiltrate of cells resembling monocytic precursors. Tri-lineage hematopoiesis with erythropoietic predominance and mild erythrophagocytosis 
was reported. Flow cytometric studies using a myeloid panel were non-diagnostic. The lymphoma panel identified a CD3 dim, CD4 and CD8 double negative population with variable CD56 expression and partial loss of CD7.

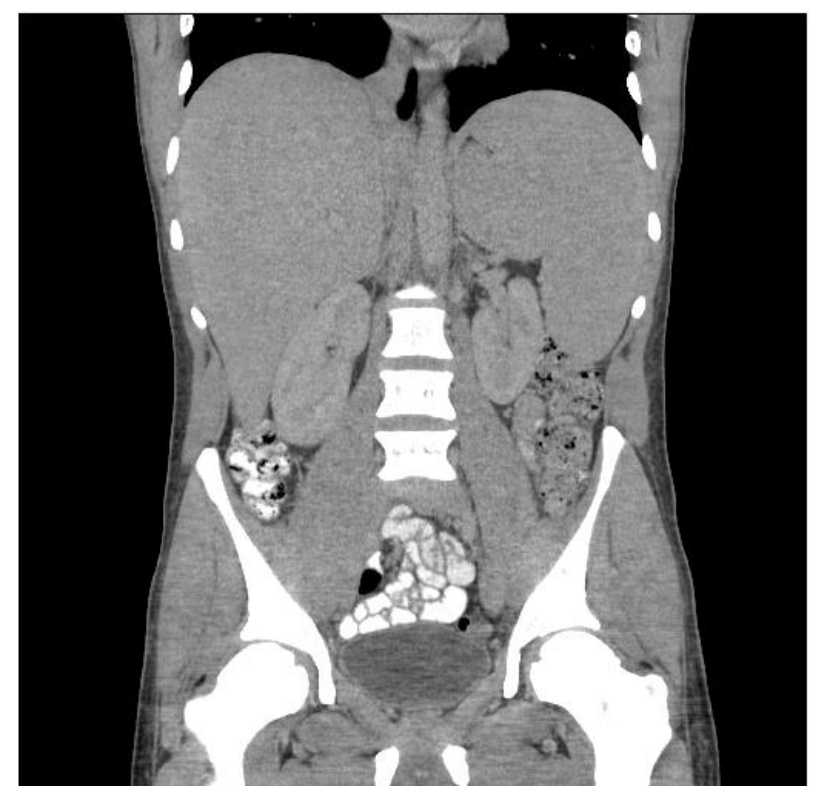

Figure 1: Computer Tomography of abdomen and pelvis demonstrating hepatosplenomegaly.

Cytogenetic analysis and FISH study confirmed the presence of isochromosome 7q. The aforementioned features were considered consistent with HSTCL. Transfer of care to a hematology department within his family's local area has subsequently been facilitated, where he is currently undergoing chemotherapy.

\section{Discussion}

The risk of HSTCL becomes a pertinent consideration in patients with IBD, given the poor prognosis of the disease and the young age of the patients. Given the use of thiopurine frequently in the treatment of UC and Crohn's disease, it is important to better describe the risk of HSTCL development associated with the use of these agents.

In many of the described cases in the literature, the onset of HSTCL in the context of thiopurine use is reported to be after 5 years of use [1,3,6,10-18]. Most reported HSTCL cases in patients with IBD had an exposure to thiopurines for at least two years [6]. Some are reported to have had concomitant anti-TNF therapy, while two separate studies have looked at monotherapy with an anti-TNF and have found no association with HSTCL $[4,19]$. A case series of 23 patients with IBD who developed HSTCL describes seven cases of infliximab naïve patients [20]. Five of these patients had received azathioprine monotherapy, one received azathioprine and cyclosporine combination, and one received 6-MP.

There are a few cases where the onset of the disease has been reported to be 2-4 years into treatment with thiopurines but these patients are often actively receiving thiopurine at the time of disease onset. The case we describe is notable as the patient had been previously on azathioprine for 2 years, however the onset of HSTCL was eight months following azathioprine cessation. Given the low incidence of HSTCL in the general population, it is difficult to estimate a relative risk in IBD patients exposed to thiopurine therapy hence the literature often cites the absolute risk. Previous cohorts examined do in fact show an increased 
www.tridhascholars.org | April-2018

absolute risk. The CESAME French cohort describes a 5.3 hazard ratio of developing a lymphoproliferative disorder in patients with IBD who had received thiopurines compared to those who did not (incidence rate of 0.9 per 1000 patient-years versus 0.26 per 1000 patient-years) [21]. Kandiel et al. [4] report a 1 in 3000-4000 overall incidence of lymphoma in a cohort of 20 to 40 -year-old IBD patients exposed to thiopurines.

HSTCL has been reported with immune-modulation therapy in other autoimmune disorders as well. One case report describes onset of HSTCL in a patient with psoriasis after treatment with the anti-TNF Etanercept [6,14]. It is unclear if this patient ever had exposure to a thiopurine. More recent cases of HSTCL development following the use of azathioprine for treatment of autoimmune hepatitis have been described [21].

Given the paucity of data regarding HSTCL, there are many unanswered questions as to what are the predisposing risk factors and how risk can be minimized in clinical practice. Literature to date suggests that the majority of cases of HSTCL involve the use of thiopurines in IBD. The case reported here is unusual given the delay in the development of the disease following azathioprine cessation. This case highlights the importance of the awareness of the risk of HSTCL in young males on azathioprine even upon discontinuation of the drug.

\section{References}

1. Mole RH (1953) Whole Body Irradiation—Radiobiology or Medicine. The British Journal of Radiology 26(305): 231241.

2. Basic Kinda S, Durakovic N, Dotlic S, et al. (2013) Hepatosplenic $\alpha \beta$ T-cell lymphoma arising after long-term azathioprine therapy successfully treated with allogeneic bone marrow transplant. Leukemia \& Lymphoma 54(6): 1334-1335.

3. Ochenrider MG, Patterson DJ and Aboulafia DM (2010) Hepatosplenic T-cell lymphoma in a young man with Crohn's disease: case report and literature review. Clinical Lymphoma, Myeloma and Leukemia 10(2): 144-148.

4. Kandiel A, Fraser AG, Korelitz BI, et al. (2005) Increased risk of lymphoma among inflammatory bowel disease patients treated with azathioprine and 6-mercaptopurine. Gut 54(8): 1121-1125.

5. Kotlyar DS, Osterman MT, Diamond RH, et al. (2011) A systematic review of factors that contribute to hepatosplenic T-cell lymphoma in patients with inflammatory bowel disease. Clinical Gastroenterology and Hepatology 9(1): 3641.

6. Subramaniam K, Yeung D, Grimpen F, et al. (2014) Hepatosplenic T-cell lymphoma, immunosuppressive agents and biologicals: what are the risks? Internal Medicine Journal 44(3): 287-290.

7. Deepak P, Sifuentes H, Sherid M, et al. (2013) T-cell non-Hodgkin's lymphomas reported to the FDA AERS with tumor necrosis factor-alpha (TNF- $\alpha$ ) inhibitors: results of the REFURBISH study. The American Journal of Gastroenterology 108(1): 99.

8. Moran G, Dillon J and Green J (2008) Crohn's disease, hepatosplenic T-cell lymphoma and no biological therapy: are we barking up the wrong tree? Inflammatory Bowel Diseases 15(9): 1281-1282.

9. Wijeratne R, Teller T and Sekhon H (2009) Hepatosplenic T-cell Lymphoma following exposure to Infliximab in a patient with ulcerative colitis.: P-0021. Inflammatory Bowel Diseases 15: S11. 
www.tridhascholars.org | April-2018

10. Drini M, Prichard PJ, Brown GJ, et al. (2008) Hepatosplenic T-cell lymphoma following infliximab therapy for Crohn's disease. The Medical Journal of Australia 189(8): 464-465.

11. Thayu M, Markowitz JE, Mamula P, et al. (2005) Hepatosplenic T-cell lymphoma in an adolescent patient after immunomodulator and biologic therapy for Crohn disease. Journal of Pediatric Gastroenterology and Nutrition 40(2): 220-222.

12. Beigel F, Jürgens M, Tillack C, et al. (2009) Hepatosplenic T-cell lymphoma in a patient with Crohn's disease. Nature Reviews Gastroenterology and Hepatology 6(7): 433.

13. Grimpen F, Yeung D, Joseph J, et al. (2009) Hepatosplenic T cell lymphoma, immunosuppressive agents and biologicals. Journal of Gastroenterology and Hepatology 24: A314.

14. Navarro JT, Ribera JM, Mate JL, et al. (2003) Hepatosplenic T-gammadelta lymphoma in a patient with Crohn's disease treated with azathioprine. Leukemia \& Lymphoma 44(3): 531-533.

15. Humphreys MR, Cino M, Quirt I, et al. (2008) Long-term survival in two patients with hepatosplenic T cell lymphoma treated with interferon-alpha. Leukemia \& Lymphoma 49(7): 1420-1423.

16. Fowler S, Beyak M, Depew WT, et al. (2010) W1212 Hepatosplenic T-Cell Lymphoma in Crohn's Disease. Where Does the Risk Lie? Gastroenterology 138(5): S-675.

17. Mackey AC, Green L, Liang LC, et al. (2007) Hepatosplenic T cell lymphoma associated with infliximab use in young patients treated for inflammatory bowel disease. Journal of Pediatric Gastroenterology and Nutrition 44(2): 265-267.

18. Yabe M, Medeiros LJ, Tang G, et al. (2016) Prognostic Factors of Hepatosplenic T-cell Lymphoma. The American Journal of Surgical Pathology 40(5): 676-688.

19. Deepak P, Sifuentes H, Sherid M, et al. (2013) T-cell non-Hodgkin's lymphomas reported to the FDA AERS with tumor necrosis factor-alpha (TNF- $\alpha$ ) inhibitors: results of the REFURBISH study. The American Journal of Gastroenterology 108(1): 99.

20. Beaugerie L, Brousse N, Bouvier AM, et al. (2009) Lymphoproliferative disorders in patients receiving thiopurines for inflammatory bowel disease: a prospective observational cohort study. The Lancet 374(9701): 1617-1625.

21. Brinkert F, Arrenberg P, Krech T, et al. (2016) Two cases of hepatosplenic T-cell lymphoma in adolescents treated for autoimmune hepatitis. Pediatrics e20154245. 